\title{
Análise do número de casos e perfil das gestantes com sífilis no estado do Paraná, Brasil durante os anos de 2007 a 2017
}

Analysis of the number of cases and profile of pregnant women with syphilis in the state of Paraná, Brazil from 2007 to 2017

Análisis del número de casos y perfil de gestantes con sífilis en el estado de Paraná, Brasil de 2007 a 2017

Recebido: 24/09/2021 | Revisado: 30/09/2021 | Aceito: 02/10/2021 | Publicado: 04/10/2021

\author{
Milena Passarelli Cortez \\ ORCID: https://orcid.org/0000-0001-5545-9810 \\ Universidade Estadual do Norte do Paraná, Brasil \\ E-mail: milenapcortez@hotmail.com \\ Daniel Augusto da Silva \\ ORCID: https://orcid.org/0000-0002-2716-6700 \\ Universidade Estadual do Norte do Paraná, Brasil \\ E-mail: daniel.augusto@uenp.edu.br \\ Emiliana Cristina Melo \\ ORCID: https://orcid.org/0000-0003-1013-4574 \\ Universidade Estadual do Norte do Paraná, Brasil \\ E-mail: ecmelo@uenp.edu.br
}

\begin{abstract}
Resumo
Objetivo: Conhecer a prevalência da sífilis gestacional no estado do Paraná, Brasil em um período de 10 anos (20072017) e o perfil epidemiológico das gestantes. Métodos: Estudo transversal e descritivo, quantitativo, no qual foi analisado os dados da sífilis gestacional no estado do Paraná, em um período de 10 anos. Foram utilizados os dados disponíveis no Departamento de Informática do Sistema Único de Saúde (DATASUS), a partir do Sistema de Informação de Agravos e Notificação (SINAN). Posteriormente, os dados foram tabulados no software Microsoft Excel ${ }^{\circledR}$ 7.0. Para o banco de dados foram selecionados os 399 municípios do estado e as variáveis ano de notificação, regional de saúde, infecção por zona de residência, faixa etária, escolaridade, raça/cor e classificação clínica. Resultados: Foi possível observar um aumento no número de casos nas 22 Regionais de Saúde, totalizando 11.450 casos em 10 anos. Quanto ao perfil das gestantes, em 18 Regionais de Saúde o perfil era de mulheres da zona urbana, com ensino fundamental incompleto, de raça/cor e branca, com faixa etária d20 a 39 anos e classificação clinica primária. Em 2 Regionais, o perfil foi de mulheres da zona urbana, com ensino fundamental incompleto, de raça/cor branca, com faixa etária de 20 a 39 anos e classificação clínica latente. Nas 2 Regionais restantes, o perfil foi de mulheres da zona urbana, com ensino fundamental incompleto, de raça/cor não branca, com faixa etária de 20 a 39 anos e classificação clínica primária. Considerações finais: Pode-se afirmar que a sífilis gestacional é uma infecção sexual transmissível que vem aumentando significativamente no estado como um todo. Foi possível observar que o perfil da maioria das gestantes infectadas era de mulheres da zona urbana, com ensino fundamental incompleto, de raça/cor branca, com faixa etária de 20 a 39 anos e com a classificação clinica primária.
\end{abstract}

Palavras-chave: Enfermagem; Epidemiologia; Infecção Sexualmente Transmissível.

\begin{abstract}
Objective: To know the prevalence of gestational syphilis in the state of Paraná, Brazil over a 10-year period (20072017) and the epidemiological profile of pregnant women. Methods: Cross-sectional and descriptive, quantitative study, in which the data of gestational syphilis in the state of Paraná in a period of 10 years was analyzed. We used the data available at the Department of Informatics of the Unified Health System (DATASUS), from the Sistema de Informação de Agravos e Notificação (SINAN). Later, the data were tabulated in Microsoft Excel® 7.0 software. For the database the 399 municipalities of the state were selected and the variables year of notification, health regional, infection by zone of residence, age group, education, race/color and clinical classification. Results: It was possible to observe an increase in the number of cases in the 22 health regions, totaling 11,450 cases in 10 years. As to the profile of pregnant women, in 18 regions the profile was of women from urban areas, with incomplete elementary schooling, of race/color and white, aged 20 to 39 years, and primary clinical classification. In 2 regions, the profile was urban women, with incomplete primary education, white, aged 20 to 39, and latent clinical classification. In the remaining 2 regions, the
\end{abstract}


profile was urban women, with incomplete elementary school education, of non-white race/color, aged 20 to 39 years, and primary clinical classification. Final considerations: It can be stated that gestational syphilis is a sexually transmitted infection that has been increasing significantly in the state as a whole. It was possible to observe that the profile of most infected pregnant women was urban, with incomplete primary education, white, aged 20 to 39 years and with primary clinical classification.

Keywords: Nursing; Epidemiology; Sexually Transmitted Infection.

\section{Resumen}

Objetivo: Conocer la prevalencia de sífilis gestacional en el estado de Paraná, Brasil, en un período de 10 años (20072017) y el perfil epidemiológico de las gestantes. Métodos: Estudio transversal y descriptivo, cuantitativo, en el que se analizaron los datos de la sífilis gestacional en el estado de Paraná en un período de 10 años. Se utilizaron los datos disponibles en el Departamento de Informática del Sistema Único de Salud (DATASUS), del Sistema de Información para el Control y Notificación de Enfermedades (SINAN). Posteriormente, los datos se tabularon en el programa Microsoft Excel® 7.0. Para la base de datos se seleccionaron los 399 municipios del estado y las variables año de notificación, salud regional, infección por zona de residencia, edad, educación, raza/color y clasificación clínica. Resultados: Se ha observado un aumento del número de casos en las 22 regiones de salud, con un total de 11.450 casos en 10 años. En cuanto al perfil de las gestantes, en 18 regiones el perfil era de mujeres de zonas urbanas, con educación primaria incompleta, de raza/color y de raza blanca, con rango de edad de 20 a 39 años y clasificación clínica primaria. En 2 regiones, el perfil era de mujeres urbanas, con educación elemental incompleta, blancas, de 20 a 39 años de edad y con clasificación clínica latente. En las 2 regiones restantes, el perfil era de mujeres urbanas, con estudios primarios incompletos, de raza/color no blanca, con edades comprendidas entre los 20 y los 39 años y clasificación clínica primaria. Consideraciones finales: Se puede afirmar que la sífilis gestacional es una infección de transmisión sexual que ha ido aumentando significativamente en el conjunto del estado. Se pudo observar que el perfil de la mayoría de las embarazadas infectadas era de mujeres urbanas, con estudios primarios incompletos, de raza blanca, con edades comprendidas entre los 20 y los 39 años y con una clasificación clínica primaria.

Palabras clave: Enfermería; Epidemiología; Infecciones de transmisión sexual.

\section{Introdução}

Sabe-se que a sífilis é uma doença infecciosa e sistêmica que possui um diagnóstico fácil e um tratamento efetivo, porém, mesmo com essas características a mesma é considerada um grande problema de saúde pública. Seu agente etiológico é o Treponema pallidum e suas formas de transmissão mais comuns são através da via sexual (relação sexual desprotegida) e via vertical (de mãe para filho durante a gestação) causando a sífilis congênita. Além disso, a sífilis possui fases distintas, sendo elas a sífilis primária, secundária e terciária e períodos de latência (Cooper, Michelow, Wozniak \& Sanches, 2016; Ministério da Saúde [MS], 2010; Magalhães, Kawaguchi, Dias \& Calderon, 2016).

É estimado, segundo a Organização Mundial da Saúde, que ocorram aproximadamente 12 milhões de novos casos de sífilis na população adulta do mundo todo e $90 \%$ ocorrem em países em desenvolvimento (Lafetá, Júnior, Silveira, \& Paranaíba 2016). No Brasil, acredita-se que ocorram mais de 900 mil casos por ano, sendo que a prevalência em gestante é de 2,6\% (Magalhães et al., 2016; Campos, Araújo, Melo \& Gonçalves, 2010).

A sífilis gestacional é atualmente um grave problema de saúde pública, pois pode ocorrer a transmissão vertical. Nas gestantes não tratadas as consequências para o recém-nascido podem incluir baixo peso ao nascer, parto prematuro, aborto, natimorto ou morte perinatal nas crianças infectadas (MS, 2010; Magalhães et al., 2016). A sífilis gestacional pode ser descoberta e tratada ainda no começo da gestação através da realização de exames nas consultas de pré-natal.

Considerando a gravidade da sífilis gestacional, esse estudo tem como objetivo principal conhecer a prevalência da mesma no estado do Paraná em um período de 10 anos (2007-2017), além do perfil epidemiológico das gestantes infectadas.

\section{Metodologia}

De acordo com Pereira, Shitsuka, Parreira e Shitsuka (2018), a sociedade é beneficiada com novos conhecimentos obtidos por meio da pesquisa científica. Dessa forma, este estudo se caracteriza como transversal e descritivo, com abordagem 
quantitativa, no qual foi analisado os dados epidemiológicos da sífilis gestacional no estado do Paraná, Brasil, em um período de 10 anos, sendo de janeiro de 2007 a dezembro de 2017. O estado do Paraná está localizado na região Sul do Brasil, possui uma área de 199.298,979 km² e uma população de aproximadamente 11.516.840 habitantes (Instituto Brasileiro de Geografia e Estatística [IBGE], 2020).

Foram utilizados os dados disponíveis no Departamento de Informática do Sistema Único de Saúde (DATASUS), sendo especificamente a partir do Sistema de Informação de Agravos e Notificação (SINAN) disponíveis até janeiro de 2019. Posteriormente, os dados foram tabulados no software Microsoft Excel® 7.0. Por meio dessas informaç̃es, foi possível conhecer a prevalência da sífilis gestacional no estado do Paraná e as características das gestantes infectadas.

Para a construção do banco de dados foram selecionados os 399 municípios do estado do Paraná e as seguintes variáveis: ano de notificação, regional de saúde, infecção por zona de residência, faixa etária, escolaridade, raça/cor e classificação clínica. Assim, as variáveis puderam ser analisadas e separadas por Regional de Saúde (RS).

O estado do Paraná é dividido em 22 Regionais de Saúde, sendo elas a $1^{\mathrm{a}} \mathrm{RS}$ - Paranaguá, $2^{\mathrm{a}} \mathrm{RS}-$ Metropolitana, $3^{\mathrm{a}}$ RS - Ponta Grossa, $4^{\mathrm{a}} \mathrm{RS}$ - Irati, $5^{\mathrm{a}} \mathrm{RS}$ - Guarapuava, $6^{\mathrm{a}} \mathrm{RS}$ - União de Vitória, $7^{\mathrm{a}} \mathrm{RS}$ - Pato Branco, $8^{\mathrm{a}} \mathrm{RS}$ - Francisco Beltrão, $9^{\mathrm{a}} \mathrm{RS}$ - Foz do Iguaçu, $10^{\mathrm{a}} \mathrm{RS}$ - Cascavel, $11^{\mathrm{a}} \mathrm{RS}$ - Campo Mourão, $12^{\mathrm{a}} \mathrm{RS}$ - Umuarama, $13^{\mathrm{a}} \mathrm{RS}$ - Cianorte, $14^{\mathrm{a}} \mathrm{RS}$ - Paranavaí, $15^{\mathrm{a}} \mathrm{RS}$ - Maringá, $16^{\mathrm{a}} \mathrm{RS}$ - Apucarana, $17^{\mathrm{a}} \mathrm{RS}$ - Londrina, $18^{\mathrm{a}} \mathrm{RS}$ - Cornélio Procópio, $19^{\mathrm{a}} \mathrm{RS}$ - Jacarezinho, $20^{\mathrm{a}} \mathrm{RS}$ - Toledo, $21^{\mathrm{a}} \mathrm{RS}$ - Telêmaco Borba e $22^{\mathrm{a}} \mathrm{RS}$ - Ivaiporã.

\section{Resultados}

Analisando os dados obtidos, foi possível observar um aumento no número de casos em todas as 22 Regionais de Saúde existentes no Paraná durante os anos estudados, totalizando 11.450 casos de sífilis gestacional em um período de 10 anos no estado. Considerando que a mesma é uma doença de notificação, é possível que após a data de realização desse estudo, tenha ocorrido uma variação do número de casos.

Analisando separadamente cada regional de saúde, notou-se que as três regionais com os maiores números de casos nos anos estudados foram a $2^{\mathrm{a}} \mathrm{RS}$ - Metropolitana com 4.610 casos, seguida da $17^{\mathrm{a}} \mathrm{RS}$ - Londrina com 983 casos e da $10^{\mathrm{a}} \mathrm{RS}-$ Cascavel com 694 casos. Já as três regionais com os menores números de casos foram a $22^{\mathrm{a}} \mathrm{RS}$ - Ivaiporã com 51 casos, a $4^{\mathrm{a}}$ RS - Irati com 85 casos e a $13^{\text {a }} \mathrm{RS}$ - Cianorte com 133 casos (Tabela 1).

Tabela 1 - Número de casos de sífilis gestacional nas 22 Regionais de Saúde do Paraná (2007-2017). Bandeirantes, (PR), Brasil, 2021.

\begin{tabular}{cc}
\hline REGIONAL DE SAÚDE & NÚMERO DE CASOS DE 2007 A 2017 - N \\
\hline $1^{\mathrm{a}}$ RS - PARANAGUÁ & 365 \\
\hline $2^{\mathrm{a}} \mathrm{RS}$ - METROPOLITANA & 4610 \\
\hline $3^{\mathrm{a}} \mathrm{RS}-$ PONTA GROSSA & 686 \\
\hline $4^{\mathrm{a}} \mathrm{RS}-$ IRATI & 85 \\
\hline $5^{\mathrm{a}} \mathrm{RS}-$ GUARAPUAVA & 333 \\
\hline $6^{\mathrm{a}} \mathrm{RS}-$ UNIÃO DE VITÓRIA & 137 \\
\hline $7^{\mathrm{a}} \mathrm{RS}-$ PATO BRANCO & 238 \\
\hline $8^{\mathrm{a}} \mathrm{RS}-$ FRANCISCO BELTRÃO & 268
\end{tabular}


Research, Society and Development, v. 10, n. 13, e64101321048, 2021

(CC BY 4.0) | ISSN 2525-3409 | DOI: http://dx.doi.org/10.33448/rsd-v10i13.21048

\begin{tabular}{cc}
\hline $9^{\mathrm{a}} \mathrm{RS}-$ FOZ DO IGUAÇU & 380 \\
\hline $10^{\mathrm{a}} \mathrm{RS}-$ CASCAVEL & 694 \\
\hline $11^{\mathrm{a}} \mathrm{RS}-$ CAMPO MOURÃO & 238 \\
\hline $12^{\mathrm{a}} \mathrm{RS}-$ UMUARAMA & 186 \\
\hline $13^{\mathrm{a}} \mathrm{RS}$ - CIANORTE & 133 \\
\hline $14^{\mathrm{a}} \mathrm{RS}-$ PARANAVAÍ & 153 \\
\hline $15^{\mathrm{a}} \mathrm{RS}-$ MARINGÁ & 610 \\
\hline $16^{\mathrm{a}} \mathrm{RS}-$ APUCARANA & 313 \\
\hline $17^{\mathrm{a}} \mathrm{RS}-$ LONDRINA & 985 \\
\hline $18^{\mathrm{a}} \mathrm{RS}-\mathrm{CORNÉLIO} \mathrm{PROCÓPIO}$ & 189 \\
\hline $19^{\mathrm{a}} \mathrm{RS}-\mathrm{JACAREZINHO}$ & 159 \\
\hline $20^{\mathrm{a}} \mathrm{RS}-$ TOLEDO & 502 \\
\hline $21^{\mathrm{a}} \mathrm{RS}-\mathrm{TELÊMACO} \mathrm{BORBA}$ & 135 \\
\hline $22^{\mathrm{a}} \mathrm{RS}-$ IVAIPORÃ & 51 \\
\hline
\end{tabular}

Fonte: Sistema de Informação e Agravos de Notificação (SINAN).

Quanto ao perfil das gestantes, notou-se que em 18 Regionais de Saúde o perfil era o mesmo, sendo este de mulheres da zona urbana, com ensino fundamental incompleto, de raça/cor branca, com faixa etária de 20 a 39 anos e classificação clinica primária (Tabela 2).

Tabela 2 - Perfil das gestantes em 18 Regionais de Saúde do Paraná (2007-2017). Bandeirantes, (PR), Brasil, 2021.

\begin{tabular}{|c|c|c|c|c|c|}
\hline $\begin{array}{c}\text { REGIONAL DE } \\
\text { SAÚDE }\end{array}$ & $\begin{array}{c}\text { ZONA DE } \\
\text { RESIDÊNCIA - } \\
\text { URBANA } \\
\text { N }(\%)\end{array}$ & $\begin{array}{c}\text { ESCOLARIDADE - } \\
\text { ENSINO } \\
\text { FUNDAMENTAL } \\
\text { INCOMPLETO } \\
\text { N (\%) }\end{array}$ & $\begin{array}{c}\text { RAÇA/COR - } \\
\text { BRANCA } \\
\text { N(\%) }\end{array}$ & $\begin{array}{l}\text { FAIXA ETÁRIA } \\
\begin{array}{r}\text {-20 A } 39 \text { ANOS } \\
\text { N (\%) }\end{array}\end{array}$ & $\begin{array}{c}\text { CLASSIFICAÇÃo } \\
\text { CLÍNICA - PRIMÁRIA } \\
\text { N (\%) }\end{array}$ \\
\hline $1^{a} \mathrm{RS}$ - PARANAGUÁ & $321(87,94)$ & $178(48,76)$ & $221(60,54)$ & $268(73,42)$ & $220(60,27)$ \\
\hline $\begin{array}{c}3^{\mathrm{a}} \mathrm{RS}-\mathrm{PONTA} \\
\text { GROSSA }\end{array}$ & $640(93,29)$ & $282(41,10)$ & $521(75,94)$ & $474(69,09)$ & $426(67,34)$ \\
\hline $4^{\mathrm{a}} \mathrm{RS}-\mathrm{IRATI}$ & $41(48,26)$ & $41(48,26)$ & $67(78,82)$ & $60(70,58)$ & $46(54,11)$ \\
\hline $\begin{array}{c}5^{\mathrm{a}} \mathrm{RS}- \\
\text { GUARAPUAVA }\end{array}$ & $232(69,66)$ & $169(50,75)$ & $214(64,26)$ & $199(59,75)$ & $240(70,07)$ \\
\hline $\begin{array}{c}\text { 6 }^{\mathrm{a}} \mathrm{RS} \text { - UNIÃO DE } \\
\text { VITÓRIA }\end{array}$ & $105(76,64)$ & $44(32,11)$ & $100(72,99)$ & $99(72,26)$ & $75(54,74)$ \\
\hline $\begin{array}{c}7^{\mathrm{a}} \mathrm{RS}-\mathrm{PATO} \\
\text { BRANCO }\end{array}$ & $348(66,79)$ & $280(53,74)$ & $338(64,87)$ & $320(61,42)$ & $285(54,70)$ \\
\hline
\end{tabular}


Research, Society and Development, v. 10, n. 13, e64101321048, 2021

(CC BY 4.0) | ISSN 2525-3409 | DOI: http://dx.doi.org/10.33448/rsd-v10i13.21048

\begin{tabular}{|c|c|c|c|c|c|}
\hline $8^{\mathrm{a}} \mathrm{RS}-\mathrm{FRANCISCO}$ & $216(80,59)$ & $92(34,32)$ & $194(72,38)$ & $180(67,16)$ & $122(45,52)$ \\
\hline \multicolumn{6}{|l|}{ BELTRÃO } \\
\hline $9^{\mathrm{a}} \mathrm{RS}-\mathrm{FOZ}$ DO & $351(92,36)$ & $128(33,68)$ & $213(56,05)$ & $280(73,68)$ & $144(37,89)$ \\
\hline \multicolumn{6}{|l|}{ IGUAÇU } \\
\hline $10^{\mathrm{a}} \mathrm{RS}-\mathrm{CASCAVEL}$ & $626(90,20)$ & $256(36,88)$ & $455(65,56)$ & $479(69,02)$ & $332(47,83)$ \\
\hline $12^{\mathrm{a}} \mathrm{RS}$ - UMUARAMA & $170(91,39)$ & $57(30,64)$ & $102(54,83)$ & $118(63,44)$ & $79(42,47)$ \\
\hline $13^{\mathrm{a}} \mathrm{RS}$ - CIANORTE & $123(92,48)$ & $52(39,09)$ & $87(65,41)$ & $91(68,42)$ & $66(49,62)$ \\
\hline 14 ${ }^{\mathrm{a}} \mathrm{RS}$ - PARANAVAÍ & $144(94,11)$ & $48(31,37)$ & $87(56,86)$ & $103(67,32)$ & $97(63,39)$ \\
\hline $15^{\mathrm{a}} \mathrm{RS}$ - MARINGÁ & $586(96,06)$ & $189(30,98)$ & $354(58,03)$ & $472(77,37)$ & $388(63,60)$ \\
\hline $16^{\mathrm{a}} \mathrm{RS}-$ & $288(92,01)$ & $98(31,30)$ & $198(63,25)$ & $216(69,00)$ & $141(45,04)$ \\
\hline \multicolumn{6}{|l|}{ APUCARANA } \\
\hline $18^{\mathrm{a}} \mathrm{RS}$ - CORNÉLIO & $164(86,77)$ & $64(33,86)$ & $110(58,20)$ & $126(66,66)$ & $128(67,72)$ \\
\hline \multicolumn{6}{|l|}{ PROCÓPIO } \\
\hline $19^{\mathrm{a}} \mathrm{RS}-$ & $137(86,16)$ & $65(40,88)$ & $104(65,40)$ & $111(69,81)$ & $80(50,31)$ \\
\hline \multicolumn{6}{|l|}{ JACAREZINHO } \\
\hline $20^{\mathrm{a}} \mathrm{RS}-\mathrm{TOLEDO}$ & $435(86,65)$ & $168(33,46)$ & $292(58,16)$ & $365(72,70)$ & $210(41,83)$ \\
\hline 21ª RS - TELÊMACO & $116(85,92)$ & $57(42,22)$ & $96(71,11)$ & $86(63,70)$ & $61(45,18)$ \\
\hline BORBA & & & & & \\
\hline
\end{tabular}

Fonte: Sistema de Informação e Agravos de Notificação (SINAN).

Das 4 regionais restantes, em 2 delas, apresentadas na Tabela 3, houve divergência quanto a classificação clínica, onde o perfil das gestantes foi de mulheres da zona urbana, com ensino fundamental incompleto, de raça/cor branca, com faixa etária de 20 a 39 anos e classificação clínica latente.

Tabela 3 - Perfil das gestantes nas Regionais de Saúde Metropolitana e Londrina do Paraná (2007-2017). Bandeirantes, (PR), Brasil, 2021.

\begin{tabular}{|c|c|c|c|c|c|}
\hline $\begin{array}{c}\text { REGIONAL DE } \\
\text { SAÚDE }\end{array}$ & $\begin{array}{c}\text { ZONA DE } \\
\text { RESIDÊNCIA - } \\
\text { URBANA } \\
\text { N }(\%)\end{array}$ & $\begin{array}{c}\text { ESCOLARIDADE - } \\
\text { ENSINO } \\
\text { FUNDAMENTAL } \\
\text { INCOMPLETO } \\
\text { N }(\%)\end{array}$ & $\begin{array}{c}\text { RAÇA/COR - } \\
\text { BRANCA } \\
\text { N }(\%)\end{array}$ & $\begin{array}{l}\text { FAIXA ETÁRIA } \\
\begin{array}{r}-20 \text { A } 39 \text { ANOS } \\
\text { N }(\%)\end{array}\end{array}$ & $\begin{array}{l}\frac{\text { CLASSIFICACÃ̃o }}{\text { CLÍNICA - LATENTE }} \\
\underline{\mathbf{N}(\%)}\end{array}$ \\
\hline $\begin{array}{c}2^{\mathrm{a}} \mathrm{RS}- \\
\text { METROPOLITANA }\end{array}$ & $4378(94,96)$ & $1389(30,13)$ & $3442(74,66)$ & $3380(73,31)$ & $2493(54,07)$ \\
\hline $17^{\mathrm{a}} \mathrm{RS}$ - LONDRINA & $928(94,21)$ & $340(34,51)$ & $560(56,85)$ & $687(67,74)$ & $465(47,20)$ \\
\hline
\end{tabular}

Fonte: Sistema de Informação e Agravos de Notificação (SINAN). 
Já as outras 2 regionais, descritas na Tabela 4, mostraram que o perfil das gestantes era de mulheres da zona urbana, com ensino fundamental incompleto, de raça/cor não branca, o que difere das demais regionais, com faixa etária de 20 a 39 anos e classificação clínica primária.

Tabela 4 - Perfil das gestantes nas Regionais de Saúde Campo Mourão e Ivaiporã do Paraná (2007-2017). Bandeirantes, (PR), Brasil, 2021.

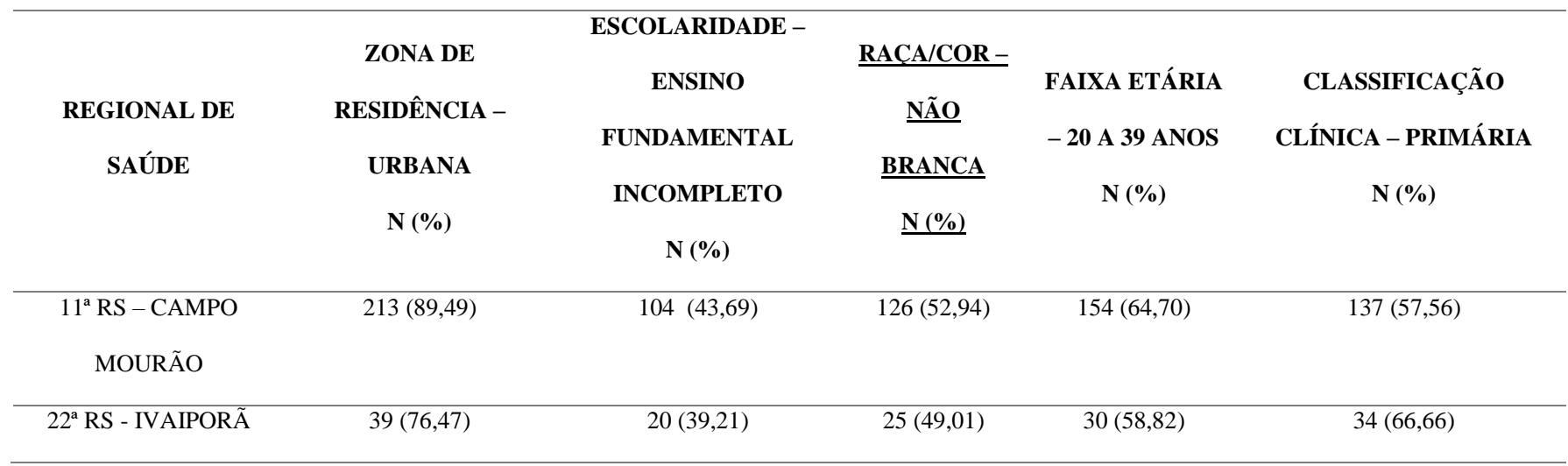

Fonte: Sistema de Informação e Agravos de Notificação (SINAN).

\section{Discussão}

Analisando a zona de residência de gestantes com sífilis em outros estados do Brasil, foi possível identificar uma concordância com os resultados obtidos em nosso estudo. Estudos realizados nos estados do Maranhão (Conceição, Câmara \& Pereira, 2019) e da Paraíba (Costa, Maia, Galisa, Almeida \& Almeida, 2021) mostram que as gestantes também eram de maioria da zona urbana.

Um estudo realizado no estado do Tocantins durante os anos de 2007 a 2017, e outro realizado no do Sergipe durante os anos de 2011 a 2015, mostram que, assim como em nosso estudo, as gestantes possuíam Ensino Fundamental Incompleto (Cavalcante, Pereira \& Castro, 2017; Andrade \& Jeraldo, 2021). Pode-se dizer que essa característica não é única do Brasil, pois um estudo realizado no Peru, mostra que as gestantes infectadas também possuíam baixa escolaridade (Gonzales, Tapia \& Serruya, 2018). É importante ressaltar que a baixa escolaridade é considerada um fator de risco para exposição às infecções sexualmente transmissíveis, já que a gestante possui um baixo entendimento sobre a importância das medidas de prevenção (Cavalcante et al., 2017).

Em relação a raça/cor das gestantes, houve prevalência da raça/cor branca, dado esse que corrobora com outros estudos realizados no estado do Paraná (Bertusso, Obregón, Moroni, Silva, Silva, Wagner \& Piazza, 2018). Analisando as duas Regionais de Saúde onde a maioria das gestantes eram da raça/cor não branca, nota-se que este resultado é também observado em âmbito nacional (Ministério da Saúde [MS], 2020).

Em relação a faixa etária das gestantes, em todas as regionais, observou-se a prevalência entre 20 a 39 anos. Estudos desenvolvidos nos Estados Unidos (Slutsker, Hennessy \& Schillinger, 2018), Paraná (Signor, Spagnolo, Tomberg, Gobatto \& Stofel, 2018) e Piauí (Campelo, BritoJúnior \& Veloso 2020) corroboram com a faixa etária predominante do nosso estudo. Esse dado pode ser justificado pois essa idade é onde as mulheres se encontram no ápice de sua fase reprodutiva (Ministério da Saúde [MS], 2016; Campos \& Campos, 2020).

Para a classificação clínica, na maioria das Regionais de Saúde, houve preponderância da sífilis primária, assim como 
em diferentes estudos (MS, 2020; Slutsker et al., 2018). Em contrapartida, assim como em um estudo realizado na cidade de Curitiba, Paraná (Moroskoski, Rozin, Batista, Queiroz \& Silva, 2018), em duas Regionais de Saúde prevaleceu a sífilis latente. Existem estudos que afirmam que a classificação clínica primária pode ser inadequada devido a impossibilidade de definir a evolução clínica da doença de forma exata (Conceição et al., 2019; MS, 2016).

\section{Considerações Finais}

Pode-se afirmar que a sífilis gestacional é uma infecção sexual transmissível que vem aumentando significativamente no estado do Paraná como um todo com o passar dos anos. Além disso, a partir desse trabalho foi possível observar também que o perfil da maioria das gestantes infectadas era de mulheres da zona urbana, com ensino fundamental incompleto, de raça/cor branca, com faixa etária de 20 a 39 anos e com a classificação clinica primária.

Como trabalhos futuros, recomenda-se novas investigações acerca do tema discutido, observando períodos e localizações diferentes. Dessa forma, será possível acompanhar a epidemiologia dos casos de sífilis gestacional para que as decisões e ações envolvendo a saúde das gestantes possam ser tomadas assertivamente.

\section{Referências}

Andrade, A. F. S. M., \& Jeraldo, V. L. S. (2021). Perfil da sífilis gestacional e congênita em Nossa Senhora do Socorro, Sergipe. Research, Society and Development, 10(2). doi: https://doi.org/10.33448/rsd-v10i2.12194

Bertusso, T. C. B., Obregón, P. L., Moroni, J. G., Silva, E. B., Silva, T. A. A. L., Wagner, L. D., \& Piazza, T. (2018). Características de gestantes com sífilis em um hospital universitário do Paraná. R. Saúde Públ, 1(2), 129-140. doi: 10.32811/25954482-2018v1n2p129

Campelo, F. S. A. A., BritoJúnior, W. V. \& Veloso, L. C. (2020). Epidemiological profile of syphilis cases in pregnant women from 2014 to 2018 in the state of Piauí. Research, Society and Development, 9(7): 1-11. doi: http://dx.doi.org/10.33448/rsd-v9i7.4382

Campos, A. L. A., Araújo, M. A. L., Melo, S. P., \& Gonçalves, M. L. C. (2010). Epidemiologia da sífilis gestacional em Fortaleza, Ceará, Brasil: um agravo sem controle. Cad. Saúde Pública, 26(9), 1747-1755. doi: 10.1590/S0102-311X2010000900008

Campos, C. O., Campos, C. O. (2020) Abordagem diagnóstica e terapêutica da sífilis gestacional e congênita: revisão narrativa. Rev Eletrônica Acervo Saúde, (53). doi: https://doi.org/10.25248/reas.e3786.2020

Cavalcante, P. A. M., Pereira, R. B. L., \& Castro, J. G. D. (2017). Sífilis gestacional e congênita em Palmas, Tocantins, 2007-2014. Epidemiologia e Serviços de Saúde, 26(2), 255-264. doi: 10.5123/S1679-49742017000200003

Conceição, H. N., Câmara, J. T., \& Pereira, B. M. (2019). Análise e espacial dos casos de sífilis gestacional e congênita. Saúde em Debate, 43(123), 1145-1158. doi: 10.1590/0103-1104201912313

Cooper, J. M., Michelow, I. C., Wozniak, P. S, \& Sanches, P. J. (2016). In time: the persistence of congenital syphilis in Brazil - More progress needed! Rev Paul Pediatr, 34(3), 251-253. doi: 10.1016/j.rppede.2016.06.004

Costa, A. P., Maia, J. T. S., Galisa, S. L. G., Almeida, R. M. S. \& Almeida, J. L. S. (2021). Perfil epidemiológico dos casos de Sífilis Gestacional na Paraíba, Brasil. Research, Society and Development, 10(9), 1-12. doi: 10.33448/rsd-v10i9.18020

Gonzales, G. F., Tapia, V., \& Serruya, S. J. (2018). Sífilis gestacional y factores asociados en hospitales públicos del Perú en el periodo 2000-2010. Rev. perú. med. exp. salud publica, 31(2), 211-221. Recuperado de http://www.scielo.org.pe/pdf/rins/v31n2/a04v31n2.pdf.

IBGE- Instituto Brasileiro de Geografia e Estatística. (2020). Cidades e estados - Paraná. Recuperado de https://cidades.ibge.gov.br/brasil/pr/panorama

Lafetá, K. R. G., Júnior, H. M., Silveira, M. F., \& Paranaíba, L. M. R. (2016). Sífilis materna e congênita, subnotificação e difícil controle. Rev bras epidemiol, 19(1). doi: https://doi.org/10.1590/1980-5497201600010006

Magalhães, D. M. S., Kawaguchi, I. A. P., Dias, A., \& Calderon, I. M. P. (2013). Sífilis materna e congênita: ainda um desafio. Cad. Saúde Pública, 29(6), 1109-1120. doi: 10.1590/S0102-311X2013000600008

Ministério da Saúde. (2016). Boletim epidemiológico: Sífilis. Recuperado de http://www.aids.gov.br/pt-br/pub/2016/boletim-epidemiologico-de-sifilis-2016 Ministério da Saúde. (2020). Boletim Epidemiológico: Sífilis. Recuperado de http://www.aids.gov.br/pt-br/pub/2020/boletim-sifilis-2020

Ministério da Saúde. (2010). Sífilis: Estratégias para Diagnóstico no https://bvsms.saude.gov.br/bvs/publicacoes/sifilis_estrategia_diagnostico_brasil.pdf 
Research, Society and Development, v. 10, n. 13, e64101321048, 2021

(CC BY 4.0) | ISSN 2525-3409 | DOI: http://dx.doi.org/10.33448/rsd-v10i13.21048

Moroskoski, M., Rozin, L., Batista, M. C., Queiroz, R. O., \& Silva, S. P. (2018). Perfil de gestantes adolescentes diagnosticadas com sífilis em Curitiba-PR. Rev Saúde Públ, 1(1), 47-58. doi: 10.32811/2595-4482.2018v1n1.39

Pereira, AS, Shitsuka, DM, Parreira, FJ \& Shitsuka, R. (2018). Metodologia da pesquisa científica. [e-book]. Santa Maria. Ed. UAB/NTE/UFSM. Disponível em: https://repositorio.ufsm.br/bitstream/handle/1/15824/Lic_Computacao_Metodologia-Pesquisa-Cientifica.pdf?sequence=1

Slutsker, J. S., Hennessy, R. R., \& Schillinger, J. A. (2018). Factors Contributing to Congenital Syphilis Cases - New York City, 2010-2016. MMWR Morb Mortal Wkly Rep, 67(39), 1088-1093. doi: 10.15585/mmwr.mm6739a3

Signor, M., Spagnolo, L. M. L., Tomberg, J. O., Gobatto, M., \& Stofel, N. S. (2018). Distribuição espacial e caracterização de casos de sífilis congênita. Revista de Enfermagem UFPE, 12(2), 398-406. doi: 10.5205/1981-8963-v12i2a230522p398-406-2018 